\title{
Test Yourself Answer: right knee pain following prolonged standing
}

\author{
Pedro Morgan Loureiro Miguelote ${ }^{1}$. Leonardo Hoehl Carneiro ${ }^{2}$. Marcelo Bragança dos Reis Oliveira ${ }^{3}$. \\ Flavia Martins Costa ${ }^{1,4}$. Diogo Goulart Corrêa ${ }^{4}$
}

Received: 9 February 2021 / Revised: 2 June 2021 / Accepted: 3 June 2021 / Published online: 11 June 2021 (c) ISS 2021

Question: A 43-year-old prison guard man with right knee pain for 3 months following prolonged standing at work

Answer: A 43-year-old prison guard man with right knee pain for 3 months following prolonged standing at work

Diagnosis: Melanoma metastasis

\section{Discussion}

A radiograph of the right knee showed a lucent oval lesion in the right distal femoral epiphysis (Fig. 1a). Computed tomography (CT) revealed a mixed lesion, with lytic and blastic components, located eccentrically in the right lateral condyle (Fig. 1b). Magnetic resonance imaging (MRI) demonstrated that the lesion was predominantly hyperintense on $\mathrm{T} 1$-weighted imaging with fat saturation, associated with peripheral gadolinium-enhancement and restricted diffusion. Dynamic contrast-enhanced perfusion MRI showed areas of early intense enhancement. Surrounding bone marrow edema with adjacent soft tissue extension could also be seen (Figs. 2 and 3).

The patient was submitted to open curettage of the lesion. Microscopic examination of hematoxylin and

The case presentation can be found at doi: 10.1007/s00256-02103838-0

Pedro Morgan Loureiro Miguelote

pedro.morganlm@gmail.com

1 Department of Radiology, Federal University of Rio de Janeiro, Rua José Linhares, ${ }^{\circ} 122$, 401, Leblon, Rio de Janeiro, Rio de Janeiro 22430-220, Brazil

2 Department of Pathology, Federal University of Rio de Janeiro, Rio de Janeiro, Brazil

3 Department of Orthopedics, Federal University of Rio de Janeiro, Rio de Janeiro, Brazil

4 Department of Radiology, Clínica de Diagnóstico Por Imagem (CDPI)/DASA, Rio de Janeiro, Brazil eosin-stained sections (Fig. 4a) revealed that the lesion was an undifferentiated epithelioid neoplasm with malignant features (high mitotic index, cytologic atypia, and necrotic foci), associated with hemorrhagic foci. Immunohistochemical analysis revealed positivity for S100, HMB-45, vimentin, and SOX-10 (Fig. 4b) and was negative for AE1/AE3 and EMA. Although the hematoxylin and eosin-stained sections did not demonstrated melanin, the immunohistochemical analysis was indicative of a malignant epithelioid neoplasm with melanocytic differentiation. The differential diagnosis included metastatic melanoma and primary clear-cell bone sarcoma. The histopathological analysis also showed a mild/moderated osteoblastic reactional activity, which can explain the mineralization pattern demonstrated on CT. BRAF mutation analysis was negative. Fluorescence in situ hybridization (FISH) for EWSR 1 rearrangement was not performed.

Retrospectively, an irregular cutaneous lesion was noted on the patient's right forearm. Histopathological evaluation of this lesion revealed a primary superficial spreading of amelanotic melanoma. Positron emission tomographycomputed tomography (PET-CT) showed multiple brain and lung metastases. The patient was referred to the department of oncology and was started on chemotherapy with dacarbazine, followed by immunotherapy (ipilimumab and nivolumab). However, the disease progressed and patient passed away.

Before the histopathological analysis, the imaging evaluation led to the consideration of common epiphyseal lesions, such as chondroblastoma and giant cell tumor. However, some characteristics of the case were atypical for both of these possible diagnoses. Chondroblastomas are generally seen in young patients with open growth plates, and giant cell tumors typically have non-sclerotic margins [1]. Chondrosarcoma and bone metastasis were also considered, based on the active characteristics seen on advanced MRI, such as restricted diffusion, 
Fig. 1 a Right knee radiograph demonstrates a lucent oval lesion in the distal femur epiphysis (arrow). b CT in the bone window, reconstructed in the sagittal plane, better shows the lesion, with mixed characteristics, with peripheral sclerosis (long arrow), as well as multiple punctate blastic components (arrowhead), located eccentrically in the right lateral condyle
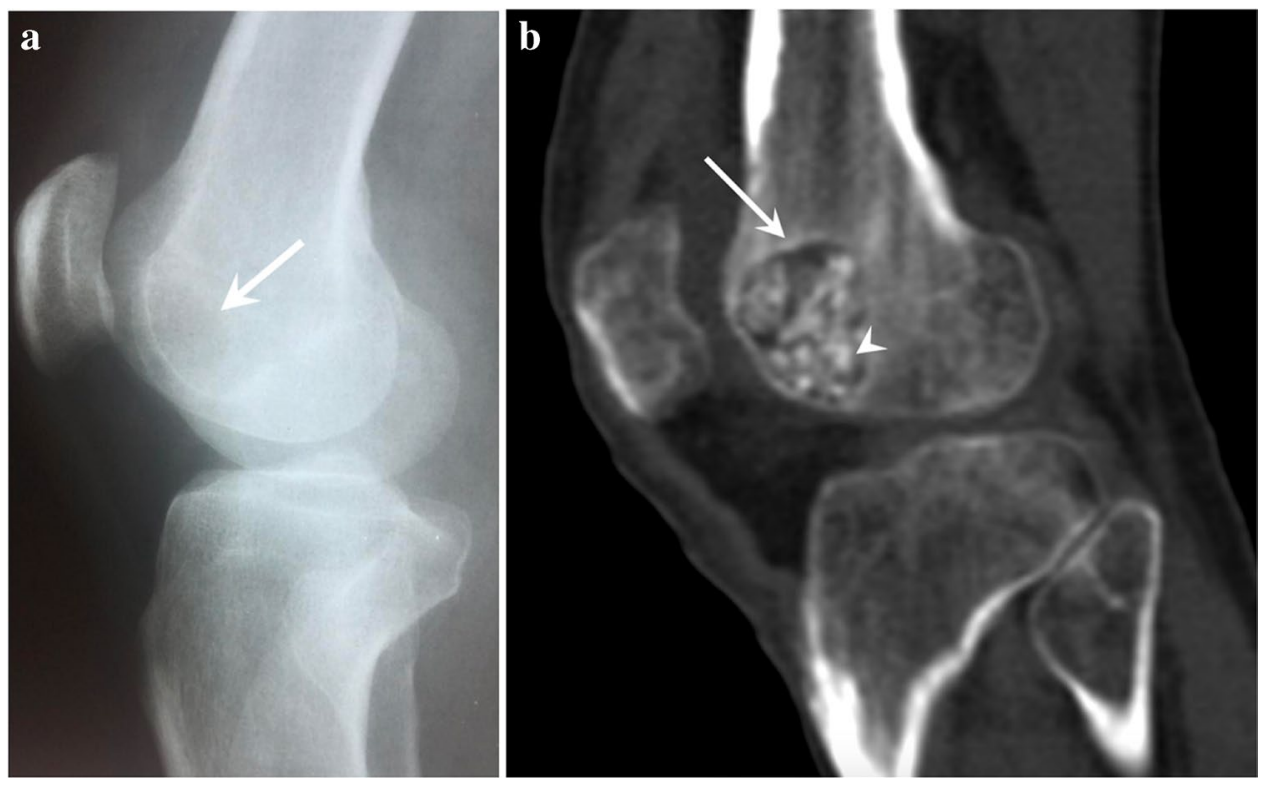

suggestive of high cellularity, and high perfusion, compatible with neoangiogenesis.

Bone metastasis of malignant melanoma is uncommon, occurring in 11-17\% of cases, predominantly in patients with advanced disease [2]. The most common clinical and laboratory findings are bone pain $(85.1 \%)$ and hypercalcemia $(14.8 \%) ; 7.4 \%$ of patients with this condition are asymptomatic at the time of diagnosis [3]. Frequent complications include spinal cord compression (20.2\%), and pathological fracture (19.1\%) [4].

Typical radiological features of melanoma bone metastasis are osteolytic lesions (87.5\%), medullary origin (91.6\%), and axial skeletal involvement $(80 \%)$. Atypical features, such as mixed osteolytic-osteoblastic components and sclerotic rim, as seen in our case, may be present in $8.2-10 \%$ and $12,5 \%$ of the cases, respectively $[2,3,5]$. The most common locations are the spine, pelvis, and ribs [3]. MRI is the modality of choice for the evaluation of soft tissue extension, and may demonstrate evidence of melanin, when it is present in sufficient quantity [6]. The femur and tibia are affected in only $7 \%$ of patients with bone metastasis from malignant melanoma, and the lesion usually arises eccentrically in the distal femoral epiphysis [3,5]. In addition, in the patient presented here, the bone metastatic lesion presented as the first clinical symptom, despite the other metastatic lesions in the lungs and brain. Although melanin in malignant melanoma may be seen on MRI, as high signal on T1-weighted images, blood products,
Fig. 2 a Right knee magnetic resonance imaging. The lesion has a predominantly hyperintense signal on T1-weighted imaging with fat-saturation (arrow). b Contrast-enhanced MRI shows areas of intense enhancement (arrow), in the periphery of the lesion, associated with central necrosis. Also, note the bone marrow edema surrounding the lesion
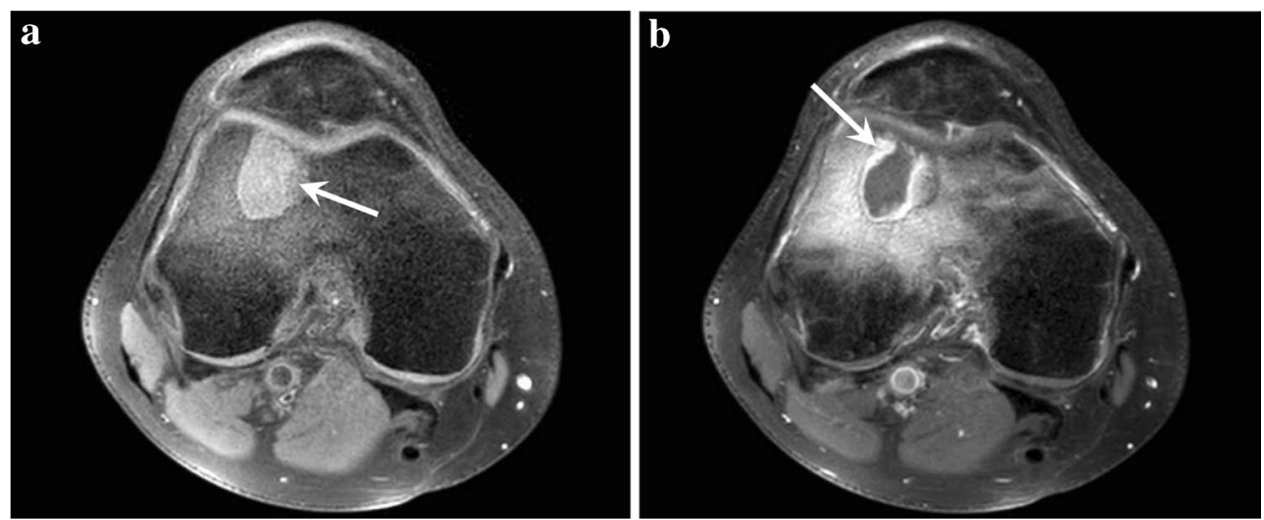
Fig. 3 Advanced magnetic resonance imaging. Diffusionweighted imaging (a) and apparent diffusion coefficient (ADC) map (b) demonstrate that the solid portion of the lesion presents restricted diffusion, due to hypercellularity. Perfusion-weighted imaging shows areas of early and rapid intense enhancement (arrow) on the color-coded semi-quantitative map (c) and time-signal intensity curve (red curve is from the solid portion of the lesion, and yellow curve is from popliteal artery), due to neoangiogenesis (d)
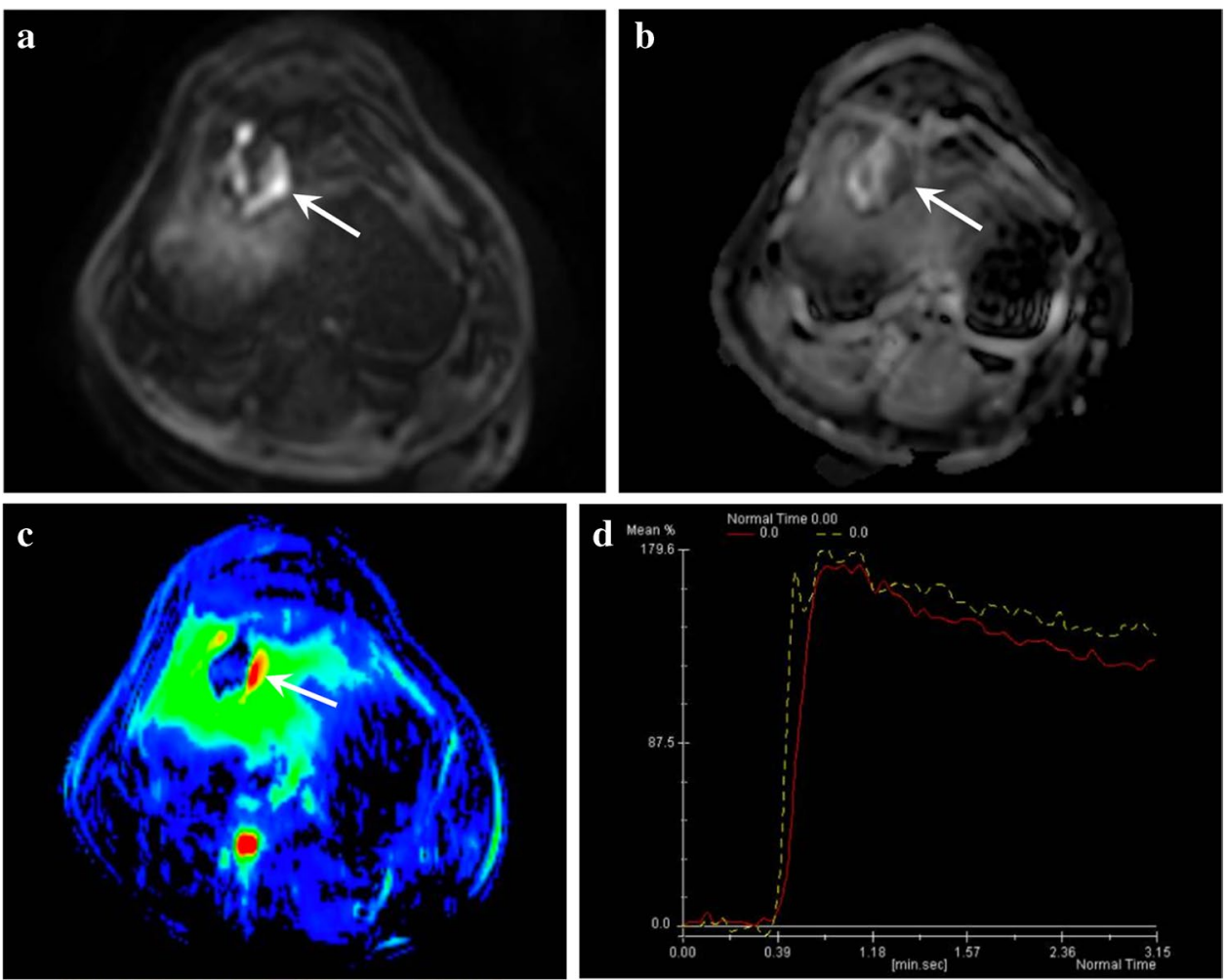

such as methemoglobin, can also contribute to the hyperintense signal on T1-weighted imaging. However, the minority of melanoma metastases presents with this typical MRI feature [7]. The histopathological analysis of our case showed an undifferentiated epithelioid neoplasm, without melanin, but with hemorrhagic foci. Therefore, the hyperintense signal on T1-weighted imaging was secondary to blood products.
Bone metastasis should be included in the differential diagnosis of epiphyseal lesions with aggressive imaging features, especially those in adults. Advanced MRI may be valuable to evaluate certain aggressive features of these tumors, such as diffusion restriction and high perfusion, particularly in cases that present atypically on conventional radiographs.
Fig. 4 a Histopathological analysis. Hematoxylin and eosin stain $(\times 400)$ demonstrates a malignant epithelioid neoplasm with high mitotic index and cytologic atypia. (b) Immunohistochemistry $(\times 100)$ shows that the tumor cells are strongly immunoreactive to S100, which is suggestive of a malignant epithelioid neoplasm with melanocytic differentiation
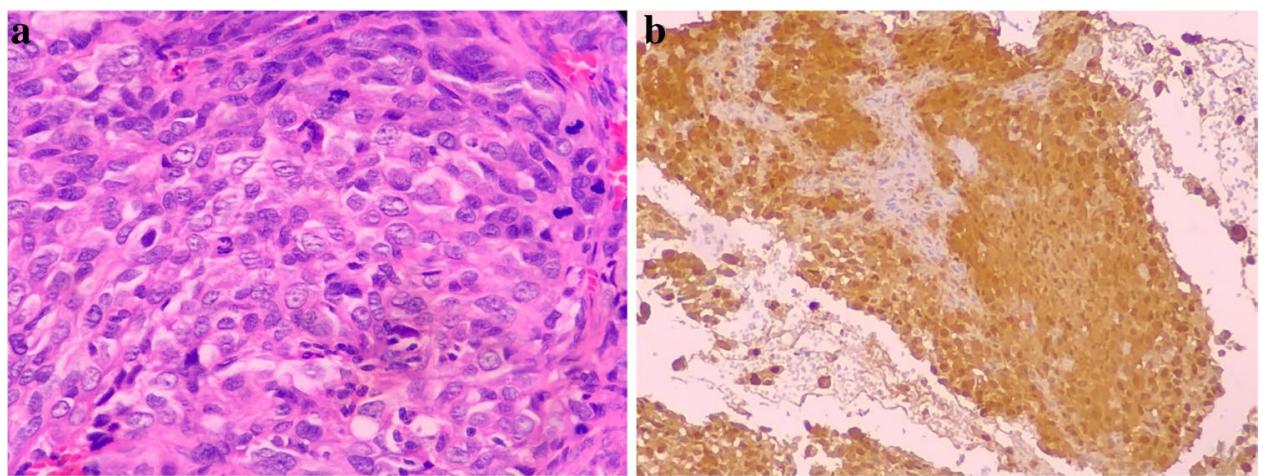


\section{Declarations}

Ethics approval All procedures performed in studies involving human participants were in accordance with ethical standards of the institutional and national research committee and with the 1964 Helsinki declaration and its later amendments or comparable ethical standards.

Informed consent Informed consent was obtained from all individual participants included in the study.

Animal rights This article does not contain any studies with animal subjects performed by the any of the authors.

Conflict of interest The authors declare no competing interests.

\section{References}

1. Chakarun CJ, Forrester DM, Gottsegen CJ, Patel DB, White EA, Matcuk GR Jr. Giant cell tumor of bone: review, mimics, and new developments in treatment. Radiographics. 2013;33(1):197-211.
2. Fishman EK, Kuhlman JE, Schuchter LM, Miller JA 3rd, Magid D. CT of malignant melanoma in the chest, abdomen, and musculoskeletal system. Radiographics. 1990;10(4):603-20.

3. Brountzos EN, Panagiotou IE, Bafaloukos D, Kelekis DA. Bone metastases from malignant melanoma: a retrospective review and analysis of 28 cases. Radiol Oncol. 2001;35:209-14.

4. Zekri J, Marples M, Taylor D, Kandukurti K, McParland L, Brown JE. Complications of bone metastases from malignant melanoma. J Bone Oncol. 2017;8:13-7.

5. Potepan P, Spagnoli I, Danesini GM, et al. The radiodiagnosis of bone metastases from melanoma. Radiol Med. 1994;87(6):741-6.

6. Patnana M, Bronstein Y, Szklaruk J, et al. Multimethod imaging, staging, and spectrum of manifestations of metastatic melanoma. Clin Radiol. 2011;66(3):224-36.

7. Escott EJ. A variety of appearances of malignant melanoma in the head: a review. Radiographics. 2001;21(3):625-39.

Publisher's note Springer Nature remains neutral with regard to jurisdictional claims in published maps and institutional affiliations. 